ARTICLE

\title{
Electrostatic melting in a single-molecule field-effect transistor with applications in genomic identification
}

Sefi Vernick1, Scott M. Trocchia ${ }^{1}$, Steven B. Warren¹, Erik F. Young ${ }^{1}$, Delphine Bouilly², Ruben L. Gonzalez², Colin Nuckolls ${ }^{2} \&$ Kenneth L. Shepard ${ }^{1}$

The study of biomolecular interactions at the single-molecule level holds great potential for both basic science and biotechnology applications. Single-molecule studies often rely on fluorescence-based reporting, with signal levels limited by photon emission from single optical reporters. The point-functionalized carbon nanotube transistor, known as the single-molecule field-effect transistor, is a bioelectronics alternative based on intrinsic molecular charge that offers significantly higher signal levels for detection. Such devices are effective for characterizing DNA hybridization kinetics and thermodynamics and enabling emerging applications in genomic identification. In this work, we show that hybridization kinetics can be directly controlled by electrostatic bias applied between the device and the surrounding electrolyte. We perform the first single-molecule experiments demonstrating the use of electrostatics to control molecular binding. Using bias as a proxy for temperature, we demonstrate the feasibility of detecting various concentrations of 20-nt target sequences from the Ebolavirus nucleoprotein gene in a constant-temperature environment.

\footnotetext{
${ }^{1}$ Department of Electrical Engineering, Columbia University, New York, New York 10027, USA. ${ }^{2}$ Department of Chemistry, Columbia University, New York, New York 10027, USA. Correspondence and requests for materials should be addressed to K.L.S. (email: shepard@ee.columbia.edu).
} 
T he study of DNA hybridization kinetics and thermodynamics is foundational to many technologies central to genomic diagnostics. Single-molecule experiments, including fluorescence-based methods such as resonance energy transfer ${ }^{1-3}$ and force-based methods ${ }^{4}$ such as atomic force microscopy $(\mathrm{AFM})^{5}$ and optical tweezers ${ }^{6}$, can be used to investigate the kinetics of association (hybridization) and dissociation (melting) of two single-stranded oligonucleotides at equilibrium ${ }^{7-11}$. While ensemble versions of such experiments are possible, single-molecule versions are much more informative ${ }^{12-14}$. The hybridization of two single-stranded oligonucleotides to form a double-stranded helix, for example, is widely analysed using a two-state model, in which formation of the double-stranded helix is driven by the favourable enthalpy of base-pairing and base-stacking interactions, and melting of the double-stranded helix is driven by the favourable configurational entropy of the single-stranded random coil.

Point-functionalized single-walled carbon nanotube (SWCNT) devices $^{15}$ have emerged as an all-electronic, label-free, singlemolecule detection platform. This single-molecule field-effect transistor (smFET) is characterized by a conductance that is sensitive to charges localized within a few Debye lengths of a point defect that is generated on the SWCNT sidewall ${ }^{16}$. This site of functionalization serves as the point of attachment of a SWCNT-tethered probe molecule under study ${ }^{17}$. Under a sourcedrain voltage bias of $100 \mathrm{mV}$, the current signal levels of a typical smFET are tens of nA. smFETs have been successfully used for the study of DNA hybridization and melting kinetics ${ }^{15}$, and DNA $^{18}$ and protein ${ }^{19,20}$ conformational dynamics.

The smFET encodes information temporally; signal amplitudes can vary as long as there is sufficient signal-to-noise ratio (SNR) contrast between the conductance states of the system. In DNA hybridization and melting experiments, the temperature is kept near the melting temperature, $T_{\mathrm{m}}$, allowing repeated hybridization and melting of the target DNA molecule to the SWCNT-tethered probe DNA molecule ${ }^{21}$. The resulting conductance versus time trajectories are used to extract target DNA concentrations, hybridization rates $\left(k_{\text {hyb }}\right)$, melting rates $\left(k_{\text {melt }}\right)$, equilibrium constant $\left(K_{\text {eq }}\right)$ and free energy of hybridization $\left(\Delta \mathrm{G}^{0}\right)^{15}$.

Probe DNA sequences of lengths $>15-20$-mer are required for practical applications in genomic identification, but these lengths present challenges in smFET studies. In particular, $T_{\mathrm{m}}$ will often exceed $60^{\circ} \mathrm{C}$, reducing the SNR (by increasing low-frequency noise and lowering the current signal ${ }^{22}$ ) and reducing device reliability (since coefficient of thermal expansion mismatches and the effect of corrosive chemical reactions increases with increasing temperature). In addition, hybridization and melting kinetics become more complex with longer probes because of the formation of relatively stable intermediates.

When measured as a function of temperature and target DNA concentrations, we find that $k_{\text {hyb }}$ exhibits non-Arrhenius behaviour that is largely unaffected by temperature (but, because it reflects a bimolecular process, is relatively sensitive to target DNA concentration), while $k_{\text {melt }}$ exhibits Arrhenius-like behaviour that it is exponentially sensitive to temperature (but, because it reflects a unimolecular process, is relatively insensitive to target DNA concentration). This electrostatic modulation of hybridization and melting kinetics allows bias to act as a proxy for temperature, with electrostatic melting (e-melting) possible at a fixed temperature, alleviating the need to operate smFET devices at elevated temperatures or as a function of temperature changes. Previous ensemble studies sometimes refer to this bias dependence from charged surfaces as electrochemical melting ${ }^{23}$, although the effects equivalent to those observed here were electrostatic in nature and did not involve charge transfer. In addition, electrostatic repulsion between the surface of the SWCNT and the probe DNA target DNA hybrid destabilizes metastable misaligned intermediates, which tend to depress $k_{\text {hyb }}$ at high target concentrations. E-melting also allows for straightforward identification of single-nucleotide polymorphisms (SNPs) in target detection through a characteristic effective melting potential, $E_{\mathrm{m}}$ (ref. 24).

\section{Results}

smFET device realization and characteristics. smFETs are based on the introduction of a point functionalization on an isolated SWCNT sidewall with, in our case, nano-confined covalent modification with a diazonium salt reagent ${ }^{18}$ (see Supplementary Note 1 and Supplementary Fig. 1). Following the isolation of individual SWCNTs, they are electrically contacted in a fieldeffect transistor configuration (see Methods) and thoroughly characterized (see Supplementary Note 2 and Supplementary Fig. 2). Post fabrication, unfunctionalized SWCNT devices are subsequently passivated with a 30-nm-thick e-beam-exposed polymethyl methacrylate (PMMA)-resist layer, leaving a 30-nm deep exposed 'window' used to confine the covalent modification (see Supplementary Note 3 and Supplementary Fig. 3).

A fabricated chip, consisting of 58 individual devices (see Supplementary Fig. 4), is stamped with a polydimethylsiloxane (PDMS) flow cell, placed directly above the isolated devices, which includes inlet and outlet ports for facile introduction of different reagents. A scaled representation of the smFET device with a tethered 20-mer probe sequence and a complementary target DNA is shown in Fig. 1a. The chip is mounted on a custom-made printed circuit board (see Supplementary Fig. 4) containing 58 independently addressable measurement channels that are simultaneously interrogated in real-time (Fig. 1a, inset). The diazonium-functionalized site in each device is conjugated to an amine-modified oligonucleotide probe. For our tethered probe DNA, we used an evolutionarily conserved sequence from the gene encoding the nucleoprotein of Zaire Ebolavirus: $5^{\prime}$-(AminoC3)-CTGTGATTTCAAATTCAGTG-3' ('Amino-C3' denotes an amino modification positioned at the $5^{\prime}$ end through a threecarbon chain linker). The target DNA sequences studied here were composed of a fully complementary 20 -mer $\left(5^{\prime}\right.$-CACTGAATTTGAAATCACAG-3'), a non-complementary 20-mer (5' GTGATTTCACTTGCAATGTC- $\left.{ }^{\prime}\right)$ and a 20-mer (5' CACTGAATTTGAAATCACAC- $3^{\prime}$ ) containing a SNP at the $-3^{\prime}$ end relative to the fully complementary 20 -mer. All experiments were performed in $43 \mathrm{mM}$ phosphate buffer at $\mathrm{pH} 8$.

Figure $1 \mathrm{~b}$ shows the variation in drain current as a function of electrolytic gate bias $\left(V_{\mathrm{g}}\right)$ at a fixed source-to-drain bias of $100 \mathrm{mV}$ for four representative smFETs before and after diazonium and subsequent DNA point functionalization. $V_{\mathrm{g}}$ is established by applying a bias through the on-chip pseudoreference platinum electrodes (fabrication and layout described in Methods and Supplementary Fig. 4). Reaction of diazonium with an $s p^{2}$-hybridized carbon atom on the surface of the SWCNT results in a covalent bond and re-hybridization of the SWCNT surface carbon atom to $s p^{3}$ (refs 25,26), reducing the conductance, as shown in Fig. 1b. Before and after functionalization, the current noise spectrum is dominated by $1 / f$ noise (flicker noise) as shown in the representative power spectral density of Fig. 1c. Following diazonium modification, the defect site dominates charge-carrier scattering rendering the rest of the SWCNT surface less sensitive to charge traps, as manifested in the reduced flicker noise. Before the introduction of the complementary 20-mer target DNA, one conductance level is evident. Upon the introduction of target DNA in solution, random telegraph noise-like transitions are observed between two discrete conductance levels, as shown in Fig. 1a, inset. These fluctuations are 


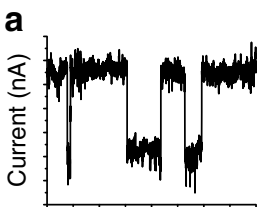

Time (s)

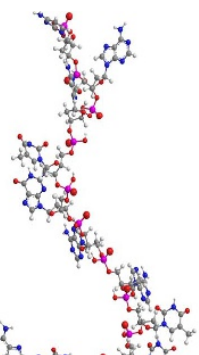

ए<smiles>[Z]1=CCCCCC1</smiles>
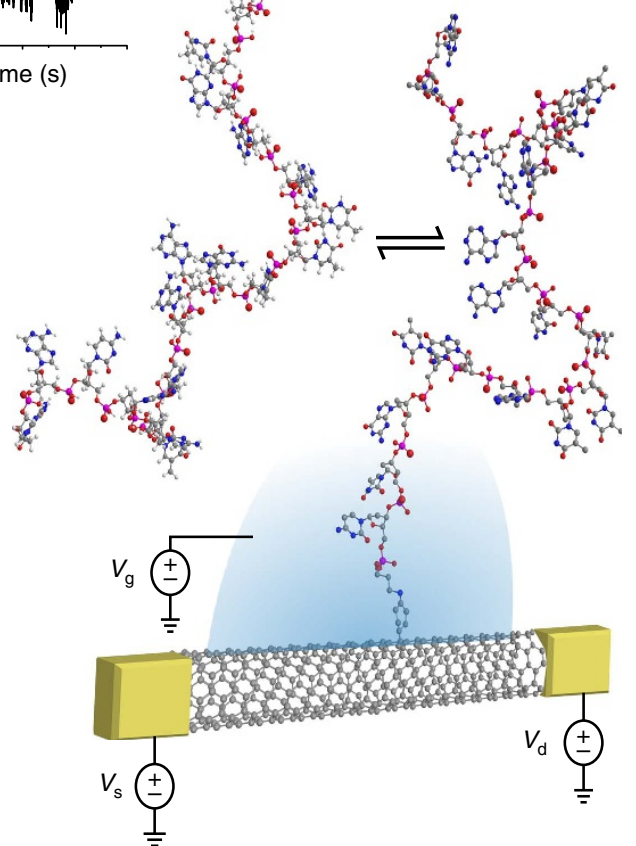

b

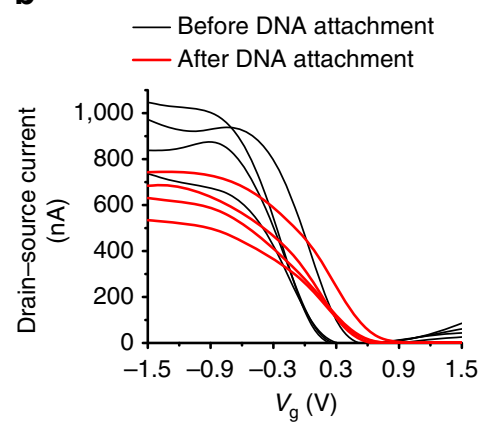

C

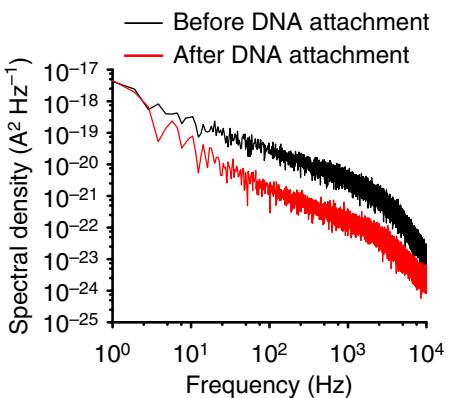

Figure 1 | smFET device characteristics. (a) To-scale illustration of the smFET device structure. An amine-modified oligonucleotide probe is covalently coupled to a single defect site, generated on a single-walled carbon nanotube (SWCNT) sidewall via diazonium chemistry. The CNT acts as the channel of a field-effect transistor, transducing biomolecular charge into a conductance change in the smFET. Binding and melting reactions of a complementary oligonucleotide generate random telegraph signals (RTSs, shown in inset), which correspond to hybridization and melting events. Experiments are performed at a constant source-drain bias $\left(V_{s}\right)$ of $100 \mathrm{mV}$, while the electrolytic gate bias is modulated by an on-chip pseudo-reference electrode. (b) Representative transfer $(I-V)$ characteristics of four CNT devices before and after point functionalization and DNA attachment. $V_{g}$ is the bias on the platinum pseudo-reference electrode relative to the device. $V_{\mathrm{g}}$ is varied from $-1.5 \mathrm{~V}$ to $+1.5 \mathrm{~V}$ (in acetonitrile solution supported by $0.1 \mathrm{M}$ tetrabutylamonium hexafluorophosphate). (c) The power spectral densities (PSDs) of the devices from $\mathbf{b}$ showing a strong flicker (1/f) noise component before (black line) and after (red line) modification. While the newly formed defect dominates transfer characteristics, the CNT sidewall becomes less sensitive to charge traps, leading to lower flicker noise.

not observed in either a control experiment containing no target DNA or in a control experiment containing the non-complementary 20-mer target DNA (both presented in Supplementary Note 5 and Supplementary Fig. 5).

The data are analysed by using an iterative event detection algorithm ${ }^{27}$. Conductance levels in each stochastic raw trajectory are assigned to discrete states, and an idealized trace is then drawn through the state assignments (as shown in Fig. $2 \mathrm{a}$ as a function of temperature). Plots of the survival probabilities in the hybridized or melted states (survival probability plots) are constructed from the idealized trajectories, yielding the rates of transition between the two conductance states (see Methods). Normalized, representative survival probability plots are shown in Fig. $2 \mathrm{~b}$ for $100 \mathrm{nM}$ complementary 20 -mer target DNA at $50^{\circ} \mathrm{C}$, the melting temperature $\left(T_{\mathrm{m}}\right)$ for this sequence, as predicted by nearest-neighbour model ${ }^{21}$ analysis (https://www.idtdna.com/ calc/analyzer). Additional survival probability plots for 30, 40 and $60^{\circ} \mathrm{C}$ are shown in Supplementary Fig. 6. The survival probabilities in the hybridized and melted states are best described by double-exponential functions with fast and slow time constants denoted as $\tau_{\text {hyb }}^{\text {fast }}$ and $\tau_{\text {hyb }}^{\text {slow }}$, respectively, for the hybridized state and $\tau_{\text {melt }}^{\text {fast }}$ and $\tau_{\text {melt }}^{\text {slow }}$, respectively, for the melted state. Each rate constant, $k_{\text {melt }}$ and $k_{\text {hyb }}$, is calculated as the reciprocal of its corresponding time constant.
For hybridization, we interpret the two components of the double-exponential functions as reflecting competing bulk (threedimensional (3D)) and surface-based (one-dimensional (1D)) diffusion processes. Because the rates of association corresponding to $3 \mathrm{D}$ and $1 \mathrm{D}$ diffusion processes are expected to have different ligand concentration dependencies, whether $k_{\text {hyb }}^{\text {fast }}$ or $k_{\text {hyb }}^{\text {slow }}$ corresponds to the $3 \mathrm{D}$ or $1 \mathrm{D}$ diffusion processes will depend on target DNA concentration. At low target DNA concentrations (for example, 1 and $10 \mathrm{nM}$ ), we interpret $k_{\text {hyb }}^{\text {slow }}$ as corresponding to the $3 \mathrm{D}$ diffusion process, since the $1 \mathrm{D}$ diffusion process is expected to be faster at lower target DNA concentrations. Above a certain target DNA concentration, however, the 3D diffusion process is expected to be faster than the $1 \mathrm{D}$ diffusion process $(1 \mathrm{D}$ diffusion coefficients are inversely proportional to ligand concentrations ${ }^{28-30}$ ), and we interpret $k_{\text {hyb }}^{\text {fast }}$ as corresponding to the $3 \mathrm{D}$ diffusion process at $100 \mathrm{nM}$. Hereafter, $k_{\mathrm{hyb}}$ is chosen as $k_{\text {hyb }}^{\text {slow }}$ or $k_{\text {hyb }}^{\text {fast }}$ as a function of target concentration to always represent the $3 \mathrm{D}$ diffusion process. The melting process, which is also described by a double-exponential function, should not depend on target concentration, which is achieved by choosing the slow and fast melting rates for $k_{\text {melt }}$ as a function of concentration in the same manner as done for $k_{\text {hyb }}$. Physically, this is a result of the fact that surface-mode melting is slower at higher target concentrations, due to the higher concentrations of 
a

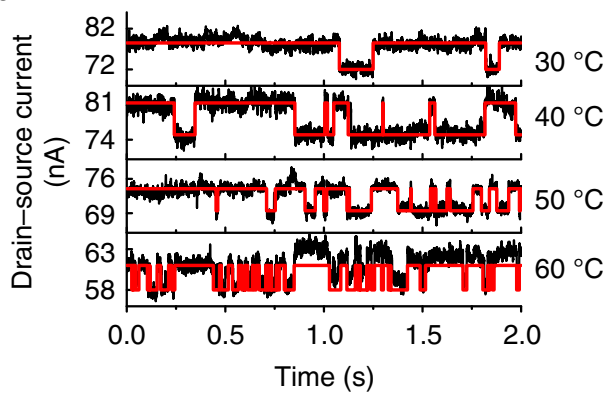

b

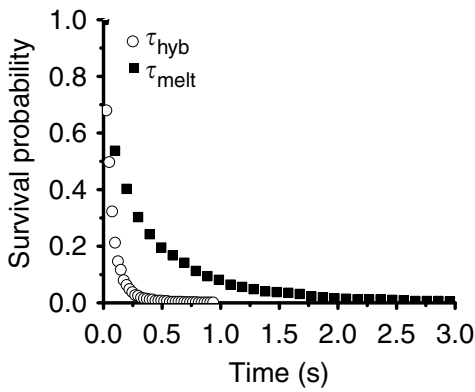

C

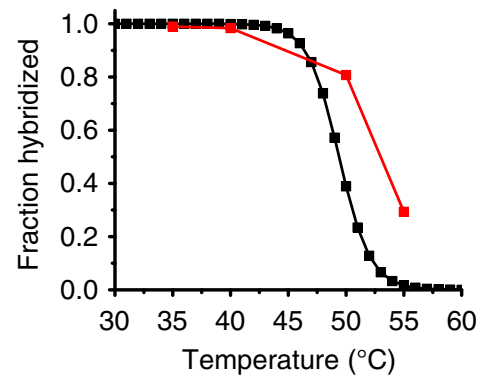

d

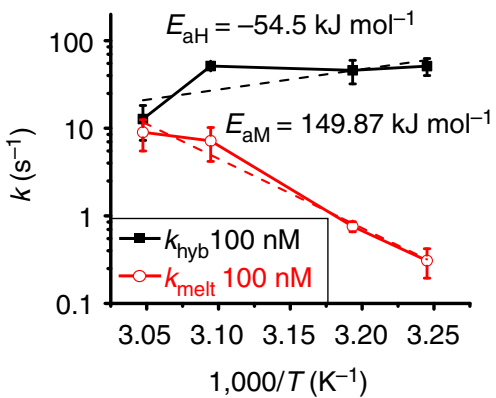

e

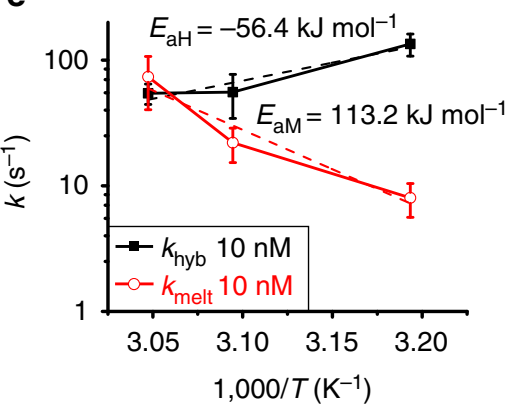

f

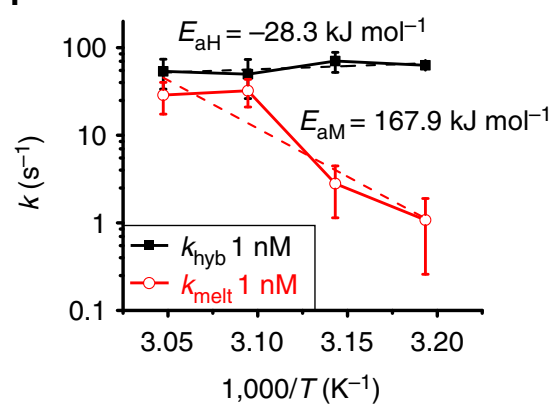

Figure 2 | Temperature-dependent DNA hybridization and corresponding data analysis. (a) Time traces of 300-s length are recorded for complementary DNA target at a concentration of $100 \mathrm{nM}$. Overlaid raw real-time data (black) and idealized fits (red) for a temperature series from 30 to $60^{\circ} \mathrm{C}$. The rate of bi-stable activity increases with temperature. (b) A representative survival probability curve for the $50^{\circ} \mathrm{C}$ trace from $\mathbf{a}$, with an event count of 763. The fit quality is characterized by $R^{2}=0.999$ and $R^{2}=0.998$ for $\tau_{\text {low } \rightarrow \text { high }}$ and $\tau_{\text {high } \rightarrow \text { low, respectively. (c) Comparison of nearest-neighbour }}$ calculated and single-molecule measured melting curves for a $100 \mathrm{nM}$ complementary target showing the same temperature dependence as a 'traditional' melting curve exhibiting a lower hybridized fraction with increasing temperature and a $T_{\mathrm{m}}$ of $52.99^{\circ} \mathrm{C}$ compared with a calculated $T_{\mathrm{m}}=49.66^{\circ} \mathrm{C}$. (d-f) Arrhenius plots for 100, 10 and $1 \mathrm{nM}$ target concentrations, showing the temperature dependence of the melting and the hybridization rates. Error bars are calculated from five different 60 -s intervals at each temperature.

surface-adsorbed negatively charged target (see Supplementary Note 7 and Supplementary Fig. 7).

Single-molecule kinetics of 20-mer oligonucleotide hybridization. Single-molecule conductance trajectories obtained for the hybridization and melting of the tethered probe DNA in the presence of $100 \mathrm{nM}$ of complementary 20-mer target DNA at various temperatures are shown in Fig. 2a. The higher-conductance state dominates at $30^{\circ} \mathrm{C}$, but this gradually shifts as a function of increasing temperature, such that the lower-conductance state dominates at $60^{\circ} \mathrm{C}$ (the same behaviour is observed over a longer time period; see Supplementary Note 8 and Supplementary Fig. 8). From the corresponding survival probability plots, shown in Fig. $2 \mathrm{~b}, k_{\mathrm{hyb}}=15.50 \mathrm{~s}^{-1}$, while $k_{\text {melt }}=15.52 \mathrm{~s}^{-1}$. Plots of the fraction of probe DNA that is hybridized as a function of temperature (that is, melting curves) for each target DNA concentration can be calculated from $1 /\left(1+k_{\text {melt }} / k_{\text {hyb }}\right)$. Figure $2 \mathrm{c}$ shows that the experimentally determined melting curve at 100 $\mathrm{nM}$ target DNA concentration (red squares and trend line), which results in a $T_{\mathrm{m}}$ of $52.99^{\circ} \mathrm{C}$, compares favourably with a predicted melting curve with a $T_{\mathrm{m}}$ of $49.66^{\circ} \mathrm{C}$, obtained using a nearest-neighbour calculation (https://www.idtdna.com/calc/ analyzer).

As shown in Fig. 2d, the temperature dependence of $k_{\text {hyb }}$ and $k_{\text {melt }}$ exhibits Arrhenius-like behaviour, with apparent activation energies $E_{\mathrm{aM}}$ and $E_{\mathrm{aH}}$ of $149.87( \pm 19.33) \mathrm{kJ} \mathrm{mol}^{-1}$ and -54.53 $( \pm 44.76) \mathrm{kJ} \mathrm{mol}^{-1}$, respectively. As expected, $k_{\text {melt }}$ shows a strong Arrhenius-like temperature dependence, while $k_{\text {hyb }}$ has a weak anti-Arrhenius behaviour, reflecting the reduced enthalpy of hydrogen bonding at elevated temperatures. At different target
DNA concentrations (Fig. 2e,f), the hybridization and melting kinetics follow a similar temperature dependence.

The target DNA concentration dependence of $k_{\text {hyb }}$ is presented in Supplementary Fig. 7b. A relatively linear target concentration dependence is observed, as expected from the diffusion-limited process. At a target concentration of $100 \mathrm{nM}$, however, $k_{\text {hyb }}$ does not appreciably increase because under these conditions, $k_{\mathrm{hyb}}$ is reaction-limited, an observation consistent with ensemble $e^{31,32}$ and single-molecule fluorescence resonance energy transfer studies $^{2}$. In addition, $k_{\text {hyb }}$ at the 1 and $10 \mathrm{nM}$ concentrations are expected to be accelerated compared to the $100 \mathrm{nM}$ concentration, since the contribution of intermediate pathways to $k_{\text {hyb }}$ becomes insignificant at these lower concentrations, again consistent with ensemble studies ${ }^{13,33,34}$.

The effect of electrostatic bias on hybridization kinetics. Temperature is often an inconvenient means to modulate $k_{\text {hyb }}$ and $k_{\text {melt }}$ in smFET-based genomic diagnostics, both because of the high temperatures that may be required to melt longer oligonucleotides and because it may not be possible to control or change temperature in large device arrays. The smFET device structure, however, provides the unique capability to modulate $k_{\text {hyb }}$ and $k_{\text {melt }}$ using an electrostatic bias between the device and the surrounding electrolyte. At an applied bias of $V_{\mathrm{g}}=0 \mathrm{~V}$, the $\mathrm{CNT}$ is not at zero charge and the reaction cannot be assumed to be solely thermally modulated. This bias point is, however, a reasonable reference point from which any additional charge would provide sufficient energy to modulate the kinetics. We find that $k_{\text {melt }}$ increases when the smFET device is placed at a negative potential relative to the surrounding electrolyte. While studies of DNA hybridization and melting kinetics (and genomic assays) 
a

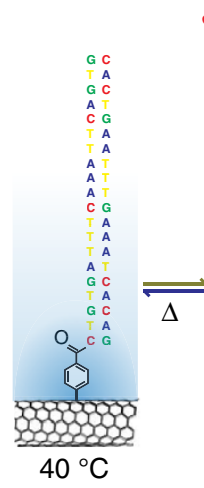

c

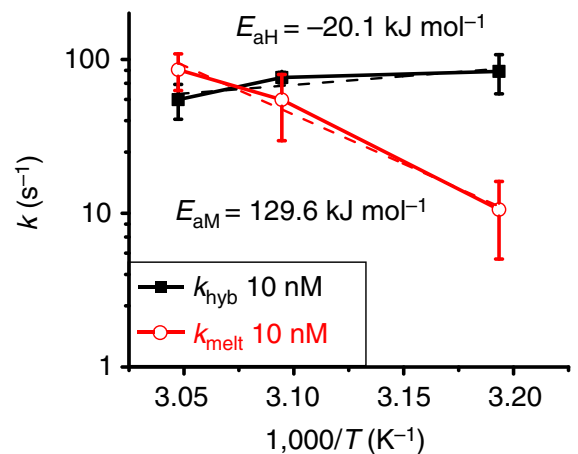

b

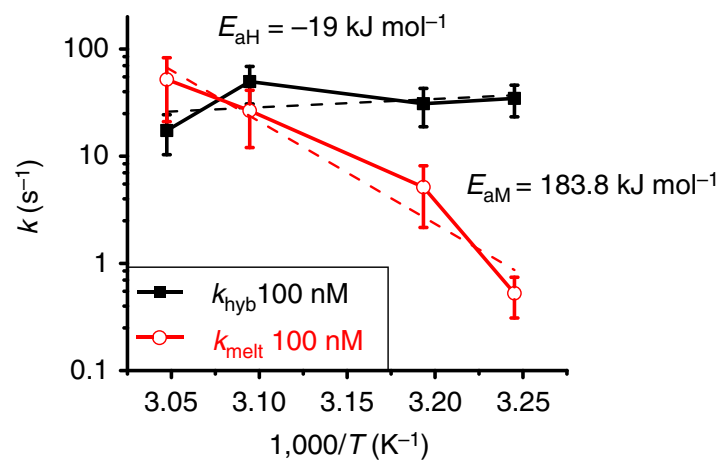

d

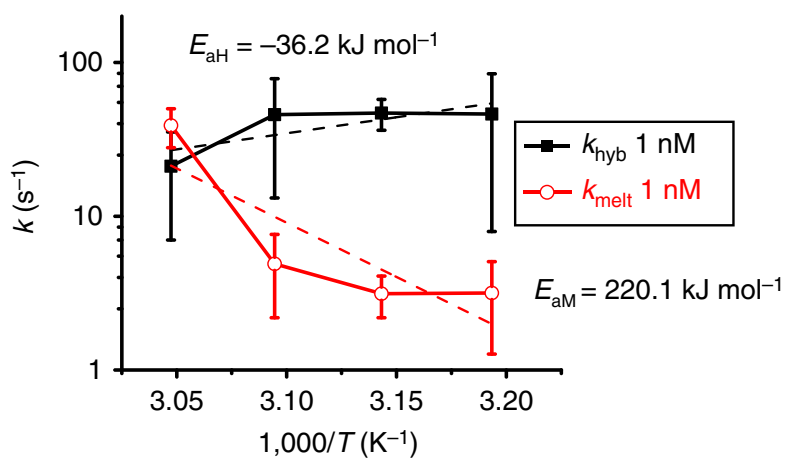

Figure 3 | The effects of bias on hybridization and melting kinetics. (a) Comparison of temperature and bias-dependent melting. With increasing temperature, the melting rate increases and hybridization rate decreases. Equivalently, when the gate bias increases, the equilibrium favours the melted state. (b-d) Arrhenius plots showing how application of gate bias affects the kinetics of melting and hybridization for 100,10 and $1 \mathrm{nM}$ complementary target. Upon the application of $300 \mathrm{mV}$ of gate bias, the entropy of activation for the melting reactions increases, while the enthalpy of activation for the hybridization reaction decreases. Error bars are calculated from five different 60-s intervals at each temperature.

a

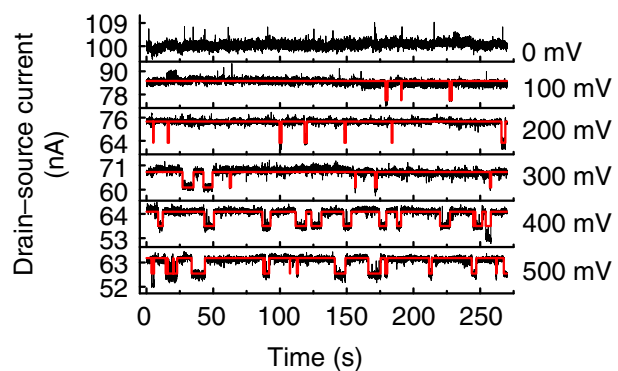

b

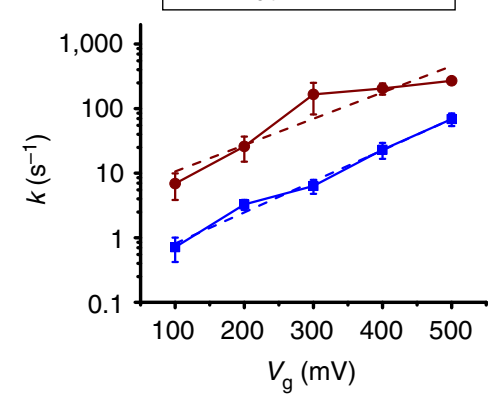

C
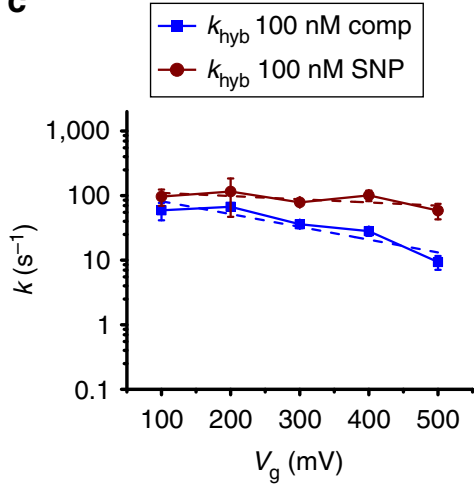

Figure 4 | Sequence dependence and the effect of e-melting on reaction kinetics. (a) Overlay of raw real-time data (black) and idealized fits (red) for a bias series ranging from $V_{g}=0 \mathrm{~V}$ to $V_{g}=500 \mathrm{mV}$ of a $100 \mathrm{nM}$ complementary target. At $0 \mathrm{~V}$, no melting events are observed and the hybridized state is dominant at $40^{\circ} \mathrm{C}$. When $V_{g}$ is increased, the melting rate increases, demonstrating longer and more frequent melting events. (b) $k_{\text {melt }} s$ of a complementary target DNA at a temperature of $40^{\circ} \mathrm{C}$ increases exponentially with increasing $V_{g}$. Behaviour of a target containing a single-base mismatch (SNP) has a noticeably smaller activation energy and higher melting rate constant at each bias point. (c) $k_{\text {hyb }}$ is less sensitive to bias. At higher bias values, $k_{\text {hyb }}$ decreases, indicating that base-pairing is affected under repulsive electrostatic force, while the SNP, which cannot pair its terminal base, does not show this effect. Error bars are calculated from five different 60 -s intervals at each temperature.

generally rely on either thermal or chemical (stringency washing) methods to modulate hybridization and melting, electrostatically driven modulation of hybridization and melting, conceived 40 years ago on macro-electrodes at the ensemble level ${ }^{35-38}$, has seen relatively little study and no single-molecule characterization.

The electrostatic force generated by the application of $300 \mathrm{mV}$ provides sufficient energy to alter $k_{\text {hyb }}$ and $k_{\text {melt }}$ compared to those observed at a $V_{\mathrm{g}}$ of $0 \mathrm{~V}$. As shown in Fig. 3a, at a negative $V_{\mathrm{g}}$, electrostatic repulsion of the negatively charged nucleotides within a Debye sphere around the SWCNT surface decreases $k_{\text {hyb }}$ and increases $k_{\text {melt }}$. (We have previously modelled this Debye screening effect in the smFET platform ${ }^{17}$ ). The temperature dependence of $k_{\text {hyb }}$ and $k_{\text {melt }}$ obtained under a $300-\mathrm{mV}$ gate bias is shown in Fig. $3 b-d$ using the complementary 20-mer 
oligonucleotide target DNA at different target DNA concentrations.

The effect of $V_{\mathrm{g}}$ on $k_{\text {hyb }}$ and $k_{\text {melt }}$ can be analysed using transition-state theory ${ }^{39}$. Specifically, $k_{\text {hyb }}$ and $k_{\text {melt }}$ as a function of temperature at a particular $V_{\mathrm{g}}$ can be fit to an equation of the form: $k_{\text {hyb (melt) }}=\left(\frac{k_{\mathrm{B}} T}{h}\right) e^{\frac{\Delta S_{\text {hyb(melt) }}^{\ddagger}}{R}} e^{\frac{-\Delta H_{\text {hyb(melt) }}^{\ddagger}}{R T}}$, where $\Delta S_{\text {hyb(melt) }}^{\ddagger}$ is the activation entropy for hybridization (melting), $\Delta H_{\mathrm{hyb} \text { (melt) }}^{\ddagger}$ is the activation enthalpy for hybridization (melting), $k_{\mathrm{B}}$ is Boltzmann's constant, $R$ is the gas constant, $h$ is Planck's constant and $\ddagger$ denotes the transition state. Although $V_{\mathrm{g}}$ affects both $k_{\text {hyb }}$ and $k_{\text {melt }}$, the strongest $V_{\mathrm{g}}$ dependence occurs for $k_{\text {melt }}$. By comparing the Arrhenius analyses of $k_{\text {melt }}$ presented in Figs $2 \mathrm{~d}-\mathrm{f}$ and $3 \mathrm{~b}-\mathrm{d}$, one finds that $\Delta S_{\text {melt }}^{\ddagger}$ increases with the application of $300-\mathrm{mV}$ bias by $115.03( \pm 62.37), 58.40( \pm 41.94)$ and $157.47( \pm 97.7)$ $\mathrm{JK}^{-1} \mathrm{~mol}^{-1}$ for 100,10 and $1 \mathrm{nM}$ target concentrations, respectively. This increase in $\Delta S_{\text {melt }}^{\ddagger}$ suggests that a disordered transition state drives a faster reaction once electrostatic bias is applied.

At the same time, the activation enthalpies of hybridization $\left(E_{\mathrm{aH}} \cong \Delta H_{\mathrm{hyb}}^{\ddagger}\right)$ become more positive with the application of bias. $\Delta H_{\text {hyb }}^{\ddagger}$ increases by $35.5,36.3$ and $7.9 \mathrm{~kJ} \mathrm{~mol}^{-1}$ for 100,10 and $1 \mathrm{nM}$ target concentrations, respectively, revealing the increased enthalpic penalty of hybridization under $300 \mathrm{mV}$ bias. This is the result of a repulsive electrostatic force that promotes melting and inhibits hybridization, eliminating the contribution of intermediate pathways to $k_{\mathrm{hyb}}$ in the absence of bias. Interestingly, we find that $k_{\text {hyb }}^{\text {slow }}$ at a target DNA concentration of $100 \mathrm{nM}$ is strongly affected by the application of an electrostatic bias, whereas $k_{\text {hyb }}$ at other target DNA concentrations are not (Supplementary Fig. 7c), suggesting that the application of electrostatic bias increases the rate of the $1 \mathrm{D}$ diffusion process at the $100 \mathrm{nM}$ target DNA concentration. This observation supports the hypothesis that the surface-based (1D) hybridization at high target DNA concentrations is decelerated due to steric interferences from other adsorbed 20-mer (see Supplementary Note 7). In the presence of electrostatic bias, this steric hindrance is mitigated by a bias that repels adsorbed DNA.

Using electrostatic bias as a proxy for temperature. The effects of $V_{\mathrm{g}}$ on the kinetics and thermodynamics of DNA hybridization and melting suggest that electrostatic modulation may be used to study hybridization and melting kinetics and thermodynamics at a fixed temperature by sweeping $V_{\mathrm{g}}$ instead of temperature. Figure $4 \mathrm{a}$ shows raw conductance trajectories and idealized hybridization and melting trajectories for one measured smFET device at a fixed temperature $\left(40^{\circ} \mathrm{C}\right)$, over a range of $V_{\mathrm{g}}$. At a $V_{\mathrm{g}}$ of $0 \mathrm{~V}$, only one conductance state, that associated with the hybridized state of the probe DNA, is apparent. With increased electrostatic bias, however, a low-conductance state associated with the melted state of the probe DNA is observed. Current histograms for each bias point are shown in Supplementary Fig. 9. Two-state transitions were not observed with the non-complementary, 20-mer target DNA (as shown by the control conductance trajectories presented in Supplementary Fig. 10).

Figure $4 \mathrm{~b}, \mathrm{c}$ shows plots of $k_{\text {melt }}$ and $k_{\text {hyb }}$ as a function of $V_{\mathrm{g}}$ for both the fully complementary 20-mer target DNA and SNPcontaining 20 -mer target DNA at a temperature of $40^{\circ} \mathrm{C}$ and $100 \mathrm{nM}$ target DNA concentration. These plots show electrostatically modulated kinetics, which bears a striking resemblance to thermally modulated kinetics. We can model this bias dependence $^{40,41}$ in $\Delta H_{\text {hyb (melt) }}^{\ddagger}$ by $\Delta H_{\text {hyb (melt) }}^{\ddagger(0)}-Q_{\text {eff }} V_{\mathrm{g}}$, where $\Delta H_{\text {hyb(melt) }}^{\ddagger(0)}$ is the hybridization (melting) activation enthalpy in the absence of bias and $Q_{\text {eff }}$ is the effective molecular charge on the target interacting with the applied field. The product of $Q_{\mathrm{eff}} V_{\mathrm{g}}$ is the reduction in the reaction energy barrier. In particular, for $k_{\text {melt }}$ of the fully complementary 20-mer target DNA, $\Delta H_{\text {melt }}^{\ddagger}=3.57( \pm 0.60) \mathrm{kJ} \mathrm{mol}^{-1}$ for a voltage-driven reaction, whereas $\Delta H_{\text {melt }}^{\ddagger(0)}=149.87( \pm 19.33) \mathrm{kJ} \mathrm{mol}^{-1}$ in absence of bias. $k_{\text {melt }}$ is larger for the SNP-containing 20-mer target DNA, as expected. In particular, $\Delta H_{\text {melt }}^{\ddagger}$ for the fully complementary 20-mer target DNA is larger compared to the SNP-containing 20-mer target DNA $\left(3.57( \pm 0.60) \mathrm{kJ} \mathrm{mol}^{-1}\right.$ compared to -2.81 $( \pm 1.84) \mathrm{kJ} \mathrm{mol}^{-1}$, respectively), a remarkable sensitivity for detecting a SNP. $k_{\text {hyb }}$ is less sensitive to electrostatic bias, as shown in Fig. 4c, providing a relationship similar to the temperature dependence of Fig. 2d. Interestingly, at higher bias values, $k_{\text {hyb }}$ for the fully complementary 20-mer target DNA decreases, indicating that pairing of the terminal base is highly sensitive to bias, whereas in the SNP-containing 20-mer target DNA, which lacks the ability to fully base pair, no such sensitivity is observed.

The Van't Hoff expression for the equilibrium constant $\left(K_{\mathrm{eq}}\right)$ governs the DNA hybridization reaction, determines the thermodynamics of the equilibrium state and is given by: $K_{\mathrm{eq}}=k_{\mathrm{hyb}} / k_{\mathrm{melt}}=e^{-\Delta G^{0} / k T}$, where $\Delta G^{0}=\Delta H^{0(0)}-T \Delta S^{0}$, where $\Delta H^{0(0)}=\Delta H_{\text {hyb }}^{\ddagger(0)}-\Delta H_{\text {melt }}^{\ddagger}$ is the enthalpy in the absence of electrostatic bias and $\Delta S^{0}=\Delta S_{\text {hyb }}^{\ddagger}-\Delta S_{\text {melt }}^{\ddagger}$. The dependence of $K_{\text {eq }}$ on temperature is shown for the fully complementary 20 -mer target DNA at a concentration of $100 \mathrm{nM}$ in Fig. 5a with and without the application of $300 \mathrm{mV}$ of $V_{\mathrm{g}}$. In the absence of electrostatic bias, an enthalpically favoured process with $\Delta H^{\circ}=-211.61( \pm 15.13) \mathrm{kJ} \mathrm{mol}^{-1}$ drives hybridization until the entropic cost becomes sufficiently high (at temperatures above $52^{\circ} \mathrm{C}$ ), as shown in Fig. 5a. The Van't Hoff plots and enthalpies obtained for 10 and $1 \mathrm{nM}$ concentrations (shown in Fig. 5b,c, respectively) also reveal large and negative enthalpies $\Delta H^{\circ}=$ $-178.50( \pm 17.58) \mathrm{kJ} \mathrm{mol}^{-1}$ for $10 \mathrm{nM}$ and $\Delta H^{\circ}=-231.19$ $( \pm 44.97) \mathrm{kJ} \mathrm{mol}^{-1}$ for $1 \mathrm{nM}$ without electrostatic bias.

The effect of electrostatic modulation of $K_{\mathrm{eq}}$ is clearly observed in Fig. $5 \mathrm{a}-\mathrm{c}$ and results from the bias dependence of the enthalpy, $\Delta H^{0}=\Delta H^{0(0)}-Q_{\text {eff }} V_{\mathrm{g}}$. When the bias is raised to $300 \mathrm{mV}$, $\Delta H^{\circ}$ increases to $-205.11( \pm 3.66) \mathrm{kJ} \mathrm{mol}^{-1}$ for the $100 \mathrm{nM}$ target DNA concentration, due to less favourable base-pairing, reducing $K_{\mathrm{eq}}$ by an order of magnitude. The reaction enthalpy is also increased at the lower target DNA concentrations (Fig. 5b,c), with $\Delta H^{\circ}=-140.04( \pm 6.92) \mathrm{kJ} \mathrm{mol}^{-1}$ and $\Delta H^{\circ}=-156.41$ $( \pm 76.42) \mathrm{kJ} \mathrm{mol}^{-1}$ for the 10 and $1 \mathrm{nM}$ target DNA concentrations, respectively.

An Arrhenius-like analysis of the plots of $K_{\mathrm{eq}}$ as a function of $V_{\mathrm{g}}$ allows one to easily extract $Q_{\text {eff }}$ and $\Delta G^{\circ}$. Changing the $5^{\prime}$ terminal base from a guanine to a cytosine in the SNPcontaining target DNA relative to the fully complementary DNA target is the most destabilizing mismatch in terms of enthalpy ${ }^{42}$, reducing the $T_{\mathrm{m}}$ by $\sim 2.6^{\circ} \mathrm{C}$. A similar change is observed in Fig. 6a, for the bias dependence of $K_{\text {eq. }}$. We can define a melting potential $\left(E_{\mathrm{m}}\right)$ as the potential at which $K_{\mathrm{eq}}=1 . E_{\mathrm{m}}$ is 400.05 $( \pm 2.53) \mathrm{mV}$ for the fully complementary target DNA, but only $326.45( \pm 1.33) \mathrm{mV}$ for the SNP-containing target DNA. $\Delta \mathrm{G}^{\circ}$ is $-15.87( \pm 1.03) \mathrm{kJ} \mathrm{mol}^{-1}$ for the fully complementary target DNA and $-9.16( \pm 1.37) \mathrm{kJ} \mathrm{mol}^{-1}$ for the SNP-containing target DNA, reflecting the fact that the hybridization reaction is more thermodynamically favourable for the fully complementary target DNA. The effective charge $\left(Q_{\text {eff }}\right)$ was previously estimated to be $0.1 \mathrm{q}$ per nucleotide ${ }^{43}$. $Q_{\text {eff }}$ associated with hybridization is slightly higher for the fully complementary target DNA in comparison with the SNP-containing target DNA $(0.40 \mathrm{q}$ compared to $0.28 \mathrm{q}$ or $\sim 4 \mathrm{nt}$ compared to $\sim 2.87 \mathrm{nt}$ ), consistent with the existence of an additional, negatively charged nucleotide closer to the surface of the SWCNT in the fully complementary 
a
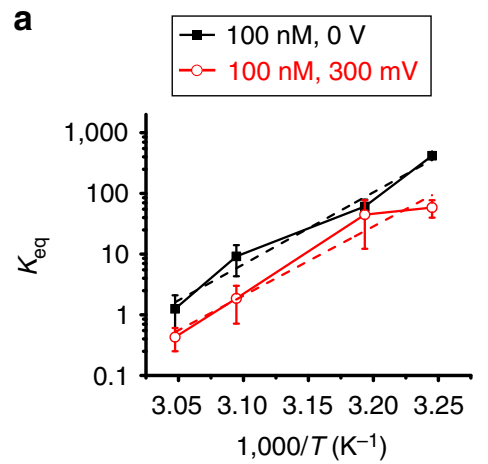

b
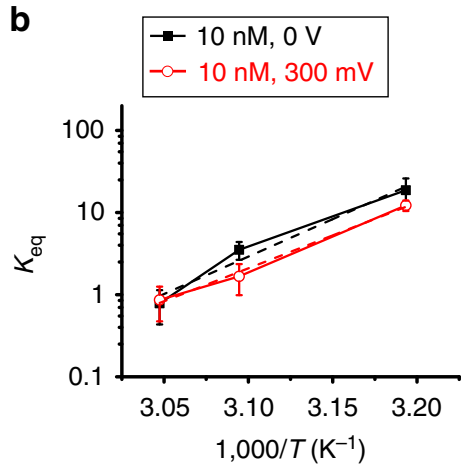

C

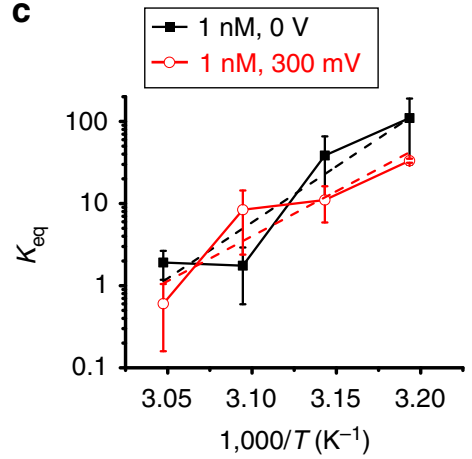

Figure 5 | Reaction thermodynamic parameters are affected by electrostatic force. Van't Hoff plots depict the effect of bias on the free energy landscape, revealing an order of magnitude lower equilibrium constants and higher enthalpy for the $300 \mathrm{mV}$ biased reaction at (a) $100 \mathrm{nM}$ complementary target, where an increase in $\Delta H^{\circ}$ of $6.5 \mathrm{~kJ} \mathrm{~mol}^{-1}$ making hybridization less favourable, (b) at $10 \mathrm{nM}$ with an enthalpy increase of $38.46 \mathrm{~kJ} \mathrm{~mol}{ }^{-1}$ and $(\mathbf{c})$ at $1 \mathrm{nM}$ with an enthalpy increase of $74.78 \mathrm{~kJ} \mathrm{~mol}^{-1}$. Error bars are calculated from five different 60 -s intervals at each temperature.

a

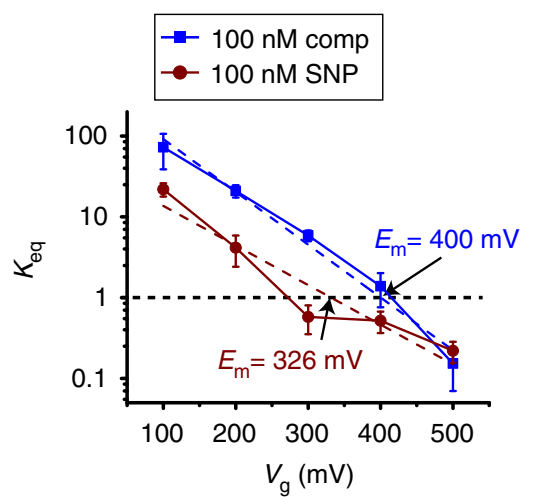

b

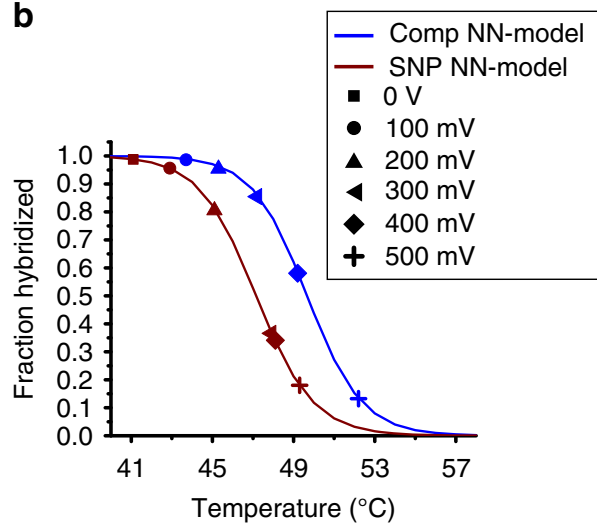

C

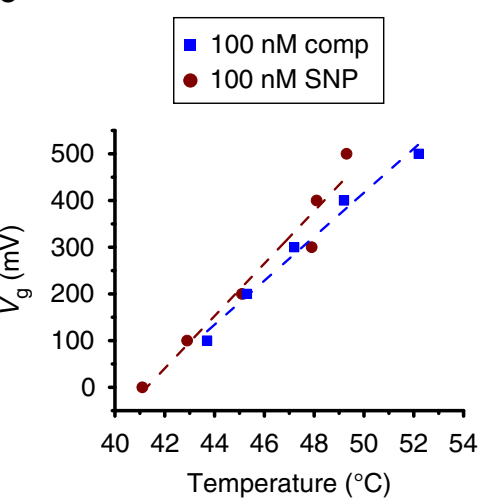

Figure 6 | Sequence-dependent effects of electrostatic force. (a) Dependence of $K_{\text {eq }}$ on $V_{g}$, demonstrating higher values for the complementary compared to the SNP across all $V_{\mathrm{g}}$ values. $K_{\text {eq }}=1$ defines a melting potential of $E_{\mathrm{m}}=400.05( \pm 2.55) \mathrm{mV}$ for the complementary target and $E_{\mathrm{m}}=326.45$ $( \pm 1.33) \mathrm{mV}$ for the SNP; $R^{2}$ values for the SNP and comp are 0.95 and 0.97 , respectively. Error bars are calculated from five different $60-\mathrm{s}$ intervals at each bias value. (b) An effective $T_{\mathrm{m}}$ curve showing the extrapolated temperatures for each hybridized fraction of both complementary and SNP target against their perspective nearest-neighbour models; and (c) calibration curves demonstrate the interchangeable effect of bias and temperature on reaction rates and further correlate each bias point with an effective temperature.

target DNA case. In all these experiments, controls employing the non-complementary 20-mer target DNAs fail to produce any two-state activity (see Supplementary Fig. 10).

Figure $6 \mathrm{~b}$ correlates the bias-dependent melting at a fixed temperature of $40^{\circ} \mathrm{C}$ to temperature-dependent melting. From a plot of the fraction of probe DNA that is hybridized versus $V_{\mathrm{g}}$, we extract an effective $T_{\mathrm{m}}$, where the fraction hybridized is 0.5 , of $49.5^{\circ} \mathrm{C}$ for the fully complementary target DNA and $47.1^{\circ} \mathrm{C}$ for the SNP-containing target DNA. The difference between these two values $\left(2.45^{\circ} \mathrm{C}\right)$ is in good agreement with the expected difference in $T_{\mathrm{m}}\left(2.6^{\circ} \mathrm{C}\right)$. With electronic control, we can influence the kinetics and thermodynamics of DNA hybridization and melting, and provide a sequence-dependent calibration between applied bias and temperature as shown in Fig. 6c. One of the mechanisms for this bias dependence may be destabilization of base-stacking, as previously hypothesized in ensemble studies $^{44}$. Our results support this explanation by observing increased activation enthalpy for hybridization upon application of negative bias.

\section{Discussion}

It is interesting to contrast these studies with surface-based ensemble assays based on hybridization, in which the number of bound target within a given area on a probe surface, usually determined fluorescently, constitutes the signal. Target concentrations, affinities and non-specific adsorption collectively affect this signal and extensive optimization is necessary to separate these factors ${ }^{45-47}$. In the single-molecule assays proposed here, however, all information is encoded temporally in the binding and unbinding kinetics ${ }^{48}$, leaving the system relatively immune to variability in signal amplitudes as long as SNR performance is maintained. smFET arrays have the same multiplexing potential as DNA microarrays but do not require any target amplification through PCR. Avoiding these the labour-intensive preparation steps required for PCR further simplifies potential analysis with label-free smFET-based single-molecule assays.

In summary, we have developed an all-electronic approach for measuring the kinetics and thermodynamics of DNA hybridization and melting as a function of temperature and electrostatic bias applied through the surrounding electrolyte. We have demonstrated that, with electronic control, we can influence the kinetics and thermodynamics of DNA hybridization and melting in a manner equivalent to temperature. An smFET array was utilized to transiently sense single-base-pair differences of two different 20-mer target DNA sequences-a fully complementary target DNA and a SNP-containing target DNA that are both derived from a gene encoded by the Zaire Ebolavirus. These 
studies pave the way for further work in the application of smFETs to genomic identification in which bias can be used as a proxy for temperature.

\section{Methods}

Device fabrication. Carbon nanotube smFET devices are constructed in the following manner. First, nanotubes are grown at $890^{\circ} \mathrm{C}$ on the surface of $1 \times 1 \mathrm{~cm}^{2}$ bare $\mathrm{Si}(500 \mu \mathrm{m}) / \mathrm{SiO}_{2}(285 \mathrm{~nm})$ die via chemical vapour deposition. The average spacing between grown nanotubes is $\sim 1$ nanotube per $100 \mu \mathrm{m}$. Second, 64 source and drain electrodes (each $8 \mathrm{~mm} \times 15 \mu \mathrm{m}$, segmented into 16 blocks of four pairs) are patterned orthogonal to the growth direction of nanotubes using a bilayer-resist photolithography process. The gap between electrodes is $4 \mu \mathrm{m}$, defining the nanotube channel length.

Titanium metal $(100 \mathrm{~nm})$ is deposited via electron-beam deposition, and the photoresist stack is lifted off. Large rectangular bars $(8 \mathrm{~mm} \times 100 \mu \mathrm{m})$ are photolithographically defined above and below the electrode pattern, and e-beam platinum $(100 \mathrm{~nm})$ is deposited to act as a pseudo-reference gate electrode. Following SEM inspection, nanotubes that bridge source-drain electrode pairs are identified. Those that transit the electrode gaps and are likely single-walled (diameter $<2 \mathrm{~nm}$, as confirmed via Raman spectroscopy and AFM characterization) are protected with a photoresist mask. All other nanotubes are etched with oxygen plasma in a Technics RIE tool ( 250 mtorr $\left.\mathrm{O}_{2}, 50 \mathrm{~W}, 12 \mathrm{~s}\right)$.

Subsequently, nanowells are patterned in a thin electron-beam resist $(950 \mathrm{~K}$ PMMA A2), in a similar manner to a formerly described method ${ }^{18}$. The resist is diluted by $25 \%$ before use to achieve a nominal layer thickness of $\sim 30 \mathrm{~nm}$. Then, it is spun at a spin speed of 8,000 r.p.m. for $1 \mathrm{~min}$. The sample is baked on a hotplate at $180^{\circ} \mathrm{C}$ for $2 \mathrm{~min}$ and loaded into a high-resolution e-beam lithography writer (Nanobeam nB4). Nanowells measuring $24 \mu \mathrm{m}$ long by $30 \mathrm{~nm}$ wide are written using $0.5-\mathrm{nA}$ beam current, thereby producing a $1: 1$ aspect ratio. The pattern is developed for $100 \mathrm{~s}$ in a cold mixture of 3:1 IPA:DI $\mathrm{H}_{2} \mathrm{O}\left(4^{\circ} \mathrm{C}\right)$, and the width of nanowells is assessed via AFM.

Diazonium functionalization. Devices are exposed to $10 \mathrm{mM}$ 4-formylbenzene diazonium hexafluorophosphate dissolved in $100 \mathrm{mM}$ sodium phosphate buffer solution with $\mathrm{pH} 8.0$ overnight on a shaking tray and in the dark. Afterwards, the thin PMMA layer is removed in heated acetone $\left(55^{\circ} \mathrm{C}\right)$ for $2 \mathrm{~h}$, rendering the surface of the chip clean again. Chips are wirebonded to ball-grid array packages using an automated wirebonder, and subsequently placed onto a custom-made circuit board described in detail below.

DNA attachment. After the functionalization stage, and after being mounted onto the circuit board, wirebonded chips are exposed to $10 \mu \mathrm{M}$ of probe DNA (Zaire Ebolavirus nucleoprotein gene, position 1,158: $5^{\prime}$-CTGTGATTTCAAATTCAGTG-3' $3^{\prime}$ in a $100 \mathrm{mM}$ sodium phosphate buffer solution with $\mathrm{pH} 8.0$, with added $200 \mu \mathrm{M}$ sodium cyanoborohydride $\left(\mathrm{NaBH}_{3} \mathrm{CN}\right)$ dissolved in $1 \mathrm{~N} \mathrm{NaOH}$, which is used to reduce the Schiff base formed between the amine and aldehyde, converting into a stable secondary amine.

Measurement set-up. The measurement set-up consists of a PDMS microfluidic channel for interfacing solution with the fully fabricated smFET devices, a custommade printed circuit board for data acquisition, and a temperature sensor/controller for fixing and modulating the temperature in the vicinity of the chip surface.

The circuit board contains 58 independently addressable measurement channels that are simultaneously interrogated in real-time. The circuitry for each channel incorporates tunable drain and source potentials, and is composed of two mutable gain stages: a front-end transimpedance amplification stage with a fixed resistive gain of $1 \mathrm{M} \Omega$, followed by an inverting voltage amplifier with variable gain from $2 \times$ to $200 \times$. Each channel, furthermore, utilizes a second-order active filter topology, limiting the signal bandwidth to $5 \mathrm{kHz}$. Readings from each channel are sampled at a rate of $25 \mathrm{kSps}$. The hardware-software interface is governed by an Opal Kelly XEM6010 FPGA module, which connects to multiplexers and analogto-digital converters on the printed circuit board, and with the PC via a USB 2.0 connection.

The PDMS microfluidic mould is constructed from a pattern drawn on a thick SU-8 layer. Such microfluidic channels have the following dimensions: 7-mm long, $750-\mu \mathrm{m}$ wide and roughly $500-\mu \mathrm{m}$ tall. Inlet and outlet holes are punched into the channel, and two sterile tubing segments are inserted. A syringe pump connected to the outlet terminal withdraws fluid exiting the channel, thus allowing full control over flow rates.

Temperature control is achieved by using a commercially available Thermostream unit capable of monitoring and modulating the temperature of forced air within a manufactured enclosure surrounding the fabricated chip and microfluidics. The temperature is allowed to reach steady state before an experimental condition is recorded.

Data analysis. Once acquired through the FPGA-to-PC interface, data are postprocessed using customizable MATLAB scripts. Local drift for $5 \mathrm{~min}$ of transient recording from each measurement channel is systematically removed. Resulting signals are low-pass filtered with a fourth-order Butterworth filter to $1 \mathrm{kHz}$ to eliminate noise close to the cutoff frequency of the anti-aliasing filter of each channel. Every trace is further analysed using an iterative event detection algorithm ${ }^{27}$, which assumes a two-state model, with wandering baseline correction. Traditionally, this single-molecule data analysis methodology is applied to evaluate current blockades due to nanopores, but the same technique can be extended to any signature with two-state random telegraph noise.

Compared to an alternate signal processing paradigm for single-molecule trajectories, the hidden Markov model, the iterative detection algorithm utilizes rudimentary statistical metrics (for example, moving average, RMS noise level) rather than Markovian matrices and machine learning principles ${ }^{49}$. Consequently, the execution speed is faster, allowing for more rapid tuning of parameters by the user. Idealized traces, resulting from fits to the raw data in the iterative detection algorithm, are used to extract single-molecule binding kinetics information. Assuming the same two-state model as before, events are classified into a 'low'- and a 'high'-conductance state. Each idealized data trace for a given experimental condition is divided into five equal parts, from which cumulative density functions are constructed for each state. Each cumulative density function is normalized to the number of event counts, thus yielding survival probability plots. Average kinetic DNA hybridization/melting rates and associated error bars are calculated from them. Algorithms for this portion of the analysis are adapted from HaMMy scripts previously written in MATLAB ${ }^{50}$.

Errors for the derived kinetic and thermodynamic parameters were calculated from the s.e.'s of the corresponding least-square weighted fits. The errors for the derived differences between thermodynamic values were combined in quadrature. The errors for parameters derived from quotients (that is, the $E_{\mathrm{m}}$, calculated by dividing the intercept by the slope) were obtained by taking the root of the sum of the squares of the fractional errors in the original quantities.

Signal specificity was further demonstrated by analysis of SNR of a two-state signature of a fully complementary strand. The dependence of SNR on gate bias, as shown in Supplementary Fig. 11, reveals an increasing trend, indicating that biasmodulated performance of the device was not responsible for the obtained signals.

Data availability. All data supporting the findings of this study are available within the article and its Supplementary Information files or from the corresponding author upon reasonable request.

\section{References}

1. Dupuis, N. F., Holmstrom, E. D. \& Nesbitt, D. J. Molecular-crowding effects on single-molecule RNA folding/unfolding thermodynamics and kinetics. Proc. Natl Acad. Sci. USA 111, 8464-8469 (2014).

2. Dupuis, N. F., Holmstrom, E. D. \& Nesbitt, D. J. Single-molecule kinetics reveal cation-promoted DNA duplex formation through ordering of single-stranded helices. Biophys. J. 105, 756-766 (2013).

3. Holmstrom, E. D., Dupuis, N. F., Nesbitt, D. J. \& Pulsed, I. R. heating studies of single-molecule DNA duplex dissociation kinetics and thermodynamics. Biophys. J. 106, 220-231 (2014).

4. Neuman, K. C. \& Nagy, A. Single-molecule force spectroscopy: optical tweezers, magnetic tweezers and atomic force microscopy. Nat. Methods 5, 491-505 (2008).

5. Williams, M. C. \& Rouzina, I. Force spectroscopy of single DNA and RNA molecules. Curr. Opin. Struc. Biol. 12, 330-336 (2002).

6. Woodside, M. T. et al. Direct measurement of the full, sequence-dependent folding landscape of a nucleic acid. Science 314, 1001-1004 (2006).

7. Wang, Q. \& Moerner, W. E. Single-molecule motions enable direct visualization of biomolecular interactions in solution. Nat. Methods 11, 555-558 (2014).

8. Schreck, J. S. et al. DNA hairpins destabilize duplexes primarily by promoting melting rather than by inhibiting hybridization. Nucleic Acids Res. 43, 6181-6190 (2015).

9. Marimuthu, K. \& Chakrabarti, R. Sequence-dependent theory of oligonucleotide hybridization kinetics. J. Chem. Phys. 140, 175104 (2014).

10. Berezhkovskii, A. M., Boguñá, M. \& Weiss, G. H. Evaluation of rate constants for conformational transitions using single-molecule fluorescence spectroscopy. Chem. Phys. Lett. 336, 321-324 (2001).

11. Dudko, O. K., Mathé, J., Szabo, A., Meller, A. \& Hummer, G. Extracting kinetics from single-molecule force spectroscopy: nanopore unzipping of DNA hairpins. Biophys. J. 92, 4188-4195 (2007).

12. Tinoco, Jr I. \& Gonzalez, Jr R. L. Biological mechanisms, one molecule at a time. Genes Dev. 25, 1205-1231 (2011).

13. Ouldridge, T. E., Sulc, P., Romano, F., Doye, J. P. \& Louis, A. A. DNA hybridization kinetics: zippering, internal displacement and sequence dependence. Nucleic Acids Res. 41, 8886-8895 (2013).

14. Correia, J. J. \& Detrich, H. W. Biophysical Tools for Biologists Vol. 1 (Academic Press, 2008). 
15. Sorgenfrei, S. et al. Label-free single-molecule detection of DNA-hybridization kinetics with a carbon nanotube field-effect transistor. Nat. Nanotechnol. 6, 125-131 (2011)

16. Goldsmith, B. R. et al. Conductance-controlled point functionalization of single-walled carbon nanotubes. Science 315, 77-81 (2007).

17. Sorgenfrei, S., Chiu C.-y., Johnston, M., Nuckolls, C. \& Shepard, K. L. Debye screening in single-molecule carbon nanotube field-effect sensors. Nano Lett. 11, 3739-3743 (2011).

18. Bouilly, D. et al. Single-molecule reaction chemistry in patterned nanowells. Nano Lett. 16, 4679-4685 (2016).

19. Choi, Y. et al. Dissecting single-molecule signal transduction in carbon nanotube circuits with protein engineering. Nano Lett. 13, 625-631 (2013).

20. Choi, Y. K. et al. Single-molecule lysozyme dynamics monitored by an electronic circuit. Science 335, 319-324 (2012).

21. Bloomfield, V. A., Crothers, D. M. \& Tinoco, I. Nucleic Acids: Structures, Properties, and Functions (University Science Books, 2000).

22. Mannik, J., Goldsmith, B. R., Kane, A. \& Collins, P. G. Chemically induced conductance switching in carbon nanotube circuits. Phys. Rev. Lett. 97, 016601 (2006).

23. Papadopoulou, E. et al. The effect of temperature on electrochemically driven denaturation monitored by SERS. Bioelectrochemistry 106, 353-358 (2015)

24. Johnson, R. P., Gao, R., Brown, T. \& Bartlett, P. N. The effect of base-pair sequence on electrochemically driven denaturation. Bioelectrochemistry $\mathbf{8 5}$, 7-13 (2012).

25. Bahr, J. L. et al. Functionalization of carbon nanotubes by electrochemical reduction of aryl diazonium salts: a bucky paper electrode. J. Am. Chem. Soc. 123, 6536-6542 (2001).

26. Schmidt, G., Gallon, S., Esnouf, S., Bourgoin, J. P. \& Chenevier, P. Mechanism of the coupling of diazonium to single-walled carbon nanotubes and its consequences. Chemestry 15, 2101-2110 (2009).

27. Plesa, C. \& Dekker, C. Data analysis methods for solid-state nanopores. Nanotechnology 26, 084003 (2015)

28. Chan, V., Graves, D. J. \& McKenzie, S. E. The biophysics of DNA hybridization with immobilized oligonucleotide probes. Biophys. J. 69, 2243-2255 (1995).

29. Chan, V., Graves, D. J., Fortina, P. \& McKenzie, S. E. Adsorption and surface diffusion of DNA oligonucleotides at liquid/solid interfaces. Langmuir 13, 320-329 (1997)

30. Chan, V., McKenzie, S. E., Surrey, S., Fortina, P. \& Graves, D. J. Effect of hydrophobicity and electrostatics on adsorption and surface diffusion of DNA oligonucleotides at liquid/solid interfaces. J. Colloid Interface Sci. 203, 197-207 (1998).

31. Erickson, D., Li, D. \& Krull, U. J. Modeling of DNA hybridization kinetics for spatially resolved biochips. Anal. Biochem. 317, 186-200 (2003).

32. Sikorav, J. L., Orland, H. \& Braslau, A. Mechanism of thermal renaturation and hybridization of nucleic acids: Kramers' process and universality in WatsonCrick base pairing. J. Phys. Chem. B 113, 3715-3725 (2009).

33. Pörschke, D. Model calculations on the kinetics of oligonucleotide double helix coil transitions. Evidence for a fast chain sliding reaction. Biophys. Chem. 2, 83-96 (1974).

34. Zhang, D. Y. \& Winfree, E. Control of DNA strand displacement kinetics using toehold exchange. J. Am. Chem. Soc. 131, 17303-17314 (2009).

35. Brabec, V. \& Paleček, E. Interaction of nucleic acids with electrically charged surfaces. Biophys. Chem. 4, 79-92 (1976).

36. Palecek, E. Normal pulse polarography of double-helical DNA - dependence of wave height on starting potential. Collect. Czech. Chem. Commun. 39, 3449-3455 (1974).

37. Palecek, E., Scheller, F. \& Wang, J. Electrochemistry of Nucleic Acids and Proteins: Towards Electrochemical Sensors for Genomics and Proteomics (Elsevier Science, 2005).

38. Valenta, P. \& Nürnberg, H. W. The electrochemical behaviour of DNA at electrically charged interfaces. Biophy. Struct. Mech. 1, 17-26 (1974).

39. Kim, J. \& Shin, J. S. Probing the transition state for nucleic acid hybridization using phi-value analysis. Biochemistry 49, 3420-3426 (2010).

40. Mathé, J., Visram, H., Viasnoff, V., Rabin, Y. \& Meller, A. Nanopore unzipping of individual DNA hairpin molecules. Biophys. J. 87, 3205-3212 (2004).

41. Nakane, J., Wiggin, M. \& Marziali, A. A nanosensor for transmembrane capture and identification of single nucleic acid molecules. Biophys. J. 87, 615-621 (2004)
42. Tikhomirova, A., Beletskaya, IV I. V. \& Chalikian, T. V. Stability of DNA Duplexes Containing GG, CC, AA, and TT Mismatches. Biochemistry 45 10563-10571 (2006).

43. Sauer-Budge, A. F., Nyamwanda, J. A., Lubensky, D. K. \& Branton, D. Unzipping kinetics of double-stranded DNA in a nanopore. Phys. Rev. Lett. 90, 238101 (2003).

44. Johnson, R. P., Gale, N., Richardson, J. A., Brown, T. \& Bartlett, P. N. Denaturation of dsDNA immobilised at a negatively charged gold electrode is not caused by electrostatic repulsion. Chem. Sci. 4, 1625-1632 (2013).

45. Gresham, D. et al. Optimized detection of sequence variation in heterozygous genomes using DNA microarrays with isothermal-melting probes. Proc. Natl Acad. Sci. USA 107, 1482-1487 (2010).

46. Suzuki, S., Ono, N., Furusawa, C., Kashiwagi, A. \& Yomo, T. Experimental optimization of probe length to increase the sequence specificity of high-density oligonucleotide microarrays. BMC Genomics 8, 373 (2007).

47. Mehlmann, M., Townsend, M. B., Stears, R. L., Kuchta, R. D. \& Rowlen, K. L. Optimization of fragmentation conditions for microarray analysis of viral RNA Anal. Biochem. 347, 316-323 (2005).

48. Makarov, D. E. Single Molecule Science (CRC Press, 2015).

49. Raillon, C., Granjon, P., Graf, M., Steinbock, L. J. \& Radenovic, A. Fast and automatic processing of multi-level events in nanopore translocation experiments. Nanoscale 4, 4916-4924 (2012).

50. McKinney, S. A., Joo, C. \& Ha, T. Analysis of single-molecule FRET trajectories using hidden markov modeling. Biophys. J. 91, 1941-1951 (2006)

\section{Acknowledgements}

We thank Jason Hon and Nathan Daly for their important insights and fruitful collaboration. We also acknowledge Alexandra Velian for assistance with organic synthesis. We thank Thomas Briese and W. Ian Lipkin for insightful advice on genomic diagnostics. This work was supported in part by the National Institutes of Health under Grants U19AI109761 and R01GM107417 and in part by the National Science Foundation under Grant 1353553.

\section{Author contributions}

S.V., S.M.T. and K.L.S. designed the experiments. S.V. and S.M.T. performed the experiments, device characterizations and data analysis. S.M.T. designed the supporting electronics, software and data analysis algorithms. E.F.Y. assisted with device fabrication. S.B.W. assisted with electronic measurements. S.V., S.M.T. and K.L.S. co-wrote the paper. D.B. assisted with nano-confined chemistry, C.N. assisted with diazonium reagent synthesis and characterization, and R.L.S. assisted with kinetic data interpretation. All authors reviewed the manuscript.

\section{Additional information}

Supplementary Information accompanies this paper at http://www.nature.com/ naturecommunications

Competing interests: The authors declare no competing financial interests.

Reprints and permission information is available online at http://npg.nature.com/ reprintsandpermissions/

How to cite this article: Vernick, S. et al. Electrostatic melting in a single-molecule field-effect transistor with applications in genomic identification. Nat. Commun. 8, 15450 doi: $10.1038 /$ ncomms15450 (2017)

Publisher's note: Springer Nature remains neutral with regard to jurisdictional claims in published maps and institutional affiliations.

This work is licensed under a Creative Commons Attribution 4.0 International License. The images or other third party material in this article are included in the article's Creative Commons license, unless indicated otherwise in the credit line; if the material is not included under the Creative Commons license, users will need to obtain permission from the license holder to reproduce the material. To view a copy of this license, visit http://creativecommons.org/licenses/by/4.0/

(C) The Author(s) 2017 